\title{
Autogenous hard palate mucosa: the ideal lower eyelid spacer?
}

\author{
Michael J Wearne, Charles Sandy, Geoffrey E Rose, J Pitts, J R O Collin
}

\begin{abstract}
Backgroundlaims-Raising a displaced lower eyelid frequently involves recession of the lower eyelid retractors with interposition of a "spacer," and several materials for this purpose have been described. This study reviewed the results of autogenous palatal mucosa in the treatment of lower eyelid displacement, including assessment of any donor site morbidity.

Methods-A retrospective case note review of consecutive patients treated at Moorfields Eye Hospital between 1993 and 1998. All patients underwent insertion of hard palate mucosa between the inferior border of the tarsus and the recessed conjunctiva and lower eyelid retractors. Parameters studied included the underlying diagnosis, measurements of lower lid displacement or retraction, related previous surgery, the experience of the operating surgeon, intraoperative and postoperative complications, surgical outcome, and length of follow up. The main outcome measure was the position of the lower eyelid relative to the globe in primary position of gaze.

Results-102 lower eyelids of 68 patients were included and a satisfactory lid position was achieved in $87 / 102(85 \%)$, with inadequate lengthening or significant recurrence of displacement occurring in $\mathbf{1 5}$ cases. Donor site haemorrhage requiring treatment in the early postoperative period occurred in seven patients $(10 \%)$.

Conclusion-Autogenous hard palate mucosa is an effective eyelid spacer and provides good long term support for the lower eyelid. Donor site complications are the main disadvantage, but may be minimised by attention to meticulous surgical technique and appropriate postoperative management.

(Br F Ophthalmol 2001;85:1183-1187)
\end{abstract}

Moorfields Eye

Hospital, London, UK

M J Wearne

C Sandy

G E Rose

J R O Collin

North East London Eye Partnership, London, UK

J Pitts

Correspondence to: Mr Geoffrey E Rose, Adnexal Service, Moorfields Eye Hospital, City Road, London EC1V 2PD, UK

Accepted for publication 13 December 2000
Most commonly associated with thyroid eye disease, lower eyelid displacement is associated with ocular discomfort and an unsightly appearance, and its surgical management frequently involves recessing the lower lid retractors and interposition of a "spacer" graft. A number of synthetic materials used for this purpose, such as poly(tetrafluoroethylene) ${ }^{1}$ or polyester mesh, ${ }^{2}$ and donor sclera is probably the most described "biological" spacer but, although effective in raising lower eyelids, ${ }^{3-5}$ it may be of limited availability and carries a risk of transmission of infections. Other techniques avoiding graftssuch as lower lid retractor tenotomy with intraoperative antimetabolites ${ }^{6}$ - have been shown to be less successful. Autogenous hard palate mucosa, first described for oculoplastic use in $1985,{ }^{7}$ is a spacer that provides both height and stiffness to support the lower lid, the tissue providing a good substitute for the posterior lamella of the eyelid. It has been used in the management of eyelid retraction, ${ }^{89}$ cicatricial entropion, ${ }^{10}$ relining of sockets, ${ }^{11}{ }^{12}$ and for exposed orbital implants. ${ }^{13}$ Other nonophthalmic uses include alar ${ }^{14}$ and lip ${ }^{15}$ reconstruction.

This paper reports the efficacy of palatal mucosa as a spacer in the treatment of lower eyelid displacement, with particular attention to the complications of the procedure - both at the recipient and the donor sites. Although the authors have used hard palate mucosa in upper eyelid reconstruction, these cases have not been included.

\section{Patients and methods}

The outcome for patients receiving lower eyelid hard palate mucosal grafting between 1993 and 1998 was reviewed from clinical records held at Moorfields Eye Hospital. With the head straight and the eyes in primary position, the degree of lower lid displacement was assessed by measurement of the inferior scleral show, and the vertical palpebral aperture and presence of lagophthalmos were also recorded. Details of the underlying disease, relevant previous ophthalmic surgery, surgical and postoperative complications, surgical outcome, and length of follow up were also drawn from the case notes.

All postoperative assessments were made a minimum of 3 months after surgery: A "good" surgical outcome was defined as the lower lid touching the inferior limbus and having a smooth contour; "acceptable" outcome where the contour was good and the lid within $1 \mathrm{~mm}$ of the lower limbus (either above or below); more than $1 \mathrm{~mm}$ of overcorrection or undercorrection was considered "unsatisfactory," unless a unilateral procedure had been carried out and the result was symmetrical with the unoperated side.

\section{DONOR SITE ANATOMY}

The oral epithelium is of stratified squamous type and tends to be keratinised in areas subject to friction, such as the hard palate; following grafting there is a rapid metaplasia to non-keratinised epithelium, thus limiting ocular irritation. ${ }^{16}$ Except over the horizontal plate of the palatine bone, where the oral mucosa and periosteum is separated by mucous glandular tissue, ${ }^{17}$ the lamina propria of the hard palate mucosa is tightly bound to the periosteum by a fibrous submucosa which is 


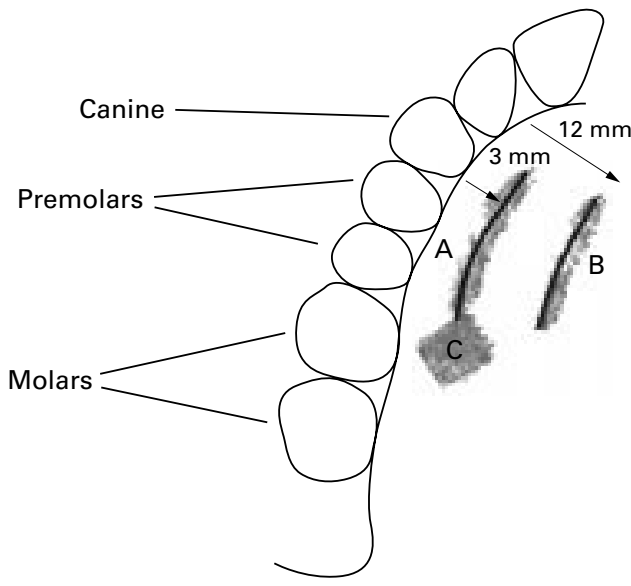

Figure 1 The thickness of palatal mucosa is greater with distance from the gingival margin, making the area " $B$ " preferable to area " $A$ " for harvesting mucosa. The mucosa is thinnest in area " $C$."

secured to the bone by Sharpey's fibres. There is no sex difference in mucosal thickness and it increases with greater distance from the marginal gingiva ${ }^{18}$ - the mucosa being thinnest near the first molar (Fig 1).

Surgeons must be completely familiar with the anatomy of the palatal donor site and be aware of the large variations in the size and shape of palatal vaults, which affect the available harvest of donor tissue; with similar horizontal palatal width, tissue availability is greater in high arched palates. ${ }^{19}$ The bones comprising the hard palate are the palatine processes of the maxillae anteriorly and the horizontal plates of the palatine bones posteriorly (Fig 2). The greater palatine neurovascular bundles emerge bilaterally from foramina of the same name and pass anteriorly along grooves in the palate, to enter the incisive foramen and pass back into the nose; veins accompany the arteries back to the pterygoid plexi. The location of this neurovascular bundle varies considerably and is most commonly found medial to the upper third molar (rather than the usual description of the upper second molar). ${ }^{19}$ In the anterior palatal region the neurovascular bundle runs towards the midline incisive canal and may be damaged if graft harvesting is not limited to the caninepremolar region.

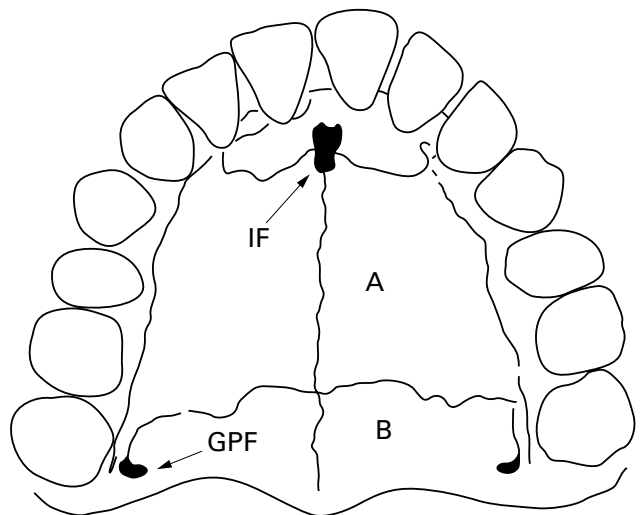

Figure 2 Osteology of the hard palate: " $A$ " maxillary. palatine processes; " $B$ " horizontal processes of the palatine bones; "IF" incisive foramen; "GPF" greater palatine foramen.

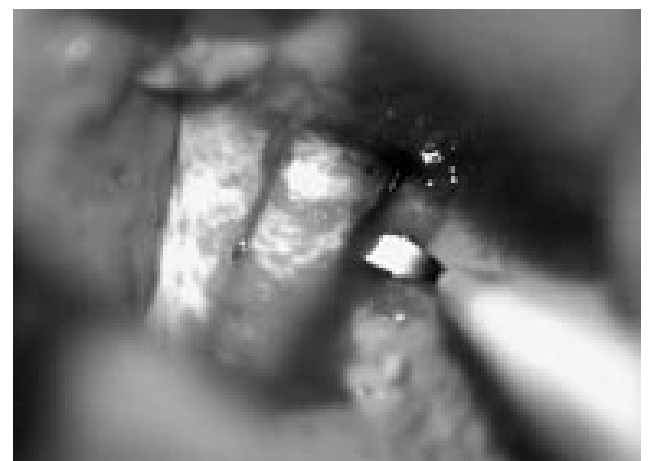

Figure 3 Hard palate mucosa being harvested, using a disposable angled keratome.

SURGICAL TECHNIQUE

The donor area was inspected for exostoses, which are not uncommon in the molar region, ${ }^{21}$ and for prominent palatal roots of teeth-the overlying mucosa often being thinner in these areas and exposure of underlying bone or roots must be avoided. Likewise, the bony grooves which house the greater palatine neurovascular bundles may be palpated, usually most easily posteriorly, to avoid incisions in the affected areas. A bifid uvula or diastasis of the soft palate can be an important clue to an underlying palatal defect that is not otherwise evident. $^{22}$

Haemostasis during graft harvesting, typically under general anaesthesia, was aided by submucosal infiltration with a $2 \%$ lignocaine and adrenaline (1:200 000) mixture and a partial thickness mucosal graft, width about twice the degree of lower lid displacement was taken using a No 15 blade or corneal pocket knife (Fig 3). Regional block in the loose areolar tissue around the greater palatine and incisive foramina may be preferred, as this avoids high pressure infiltration in the hard palate which may hydrodissect the periosteum from the bone, with risk of subperiosteal haematoma or mucosal necrosis. ${ }^{23}$

Although haemostasis is required, it is important not to bare the periosteum or apply excessive diathermy, as this may cause necrosis of the underlying bone (Fig 4). The donor site often oozes diffusely and it may be necessary to supplement cautery with placement of commercial haemostatic gauze. Brisk bleeding from the palatine vessels may be treated by injection of a local anaesthetic containing a vasoconstrictor, followed by 5 minutes of direct pressure, or it may very rarely be necessary to place encircling sutures proximal to the bleeding site. If haemostasis is still not achieved, elevation of a full thickness mucosal flap, including the vessels, allows them to be ligated under direct vision. ${ }^{24}$

An infratarsal incision, extending from below the punctum to the lateral canthus, was made through the conjunctiva and lower lid retractors, and the graft secured (using $6 / 0$ or $8 / 0$ absorbable sutures) between the lower border of tarsus and the recessed inferior retractors and conjunctiva. Postoperative care included control of pain and infection at the donor site - with systemic antibiotics, analgesic 


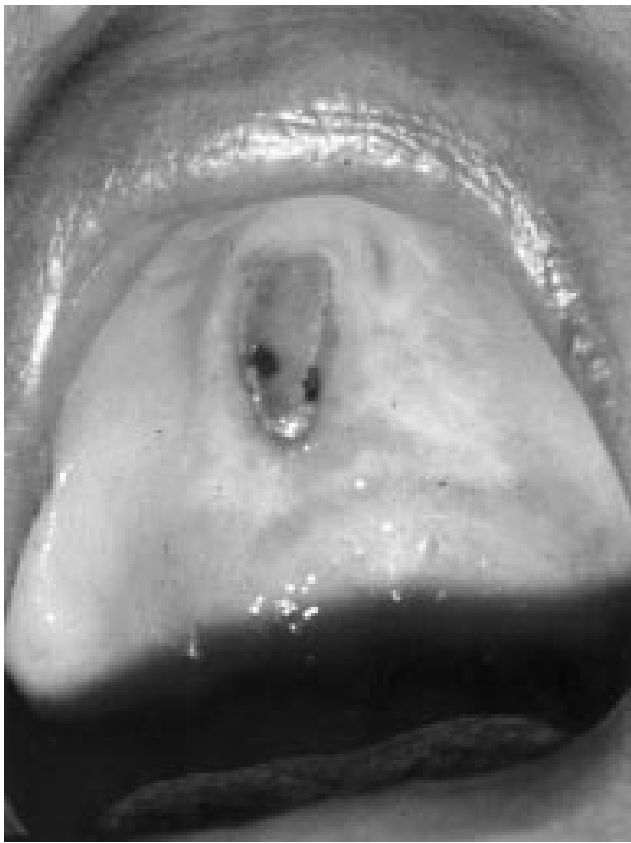

Figure 4 Oro-nasal fistula at hard palate mucosa donor site.

lozenges, and antiseptic mouth washes-and the use of local topical antibiotic drops to the recipient site.

\section{Results}

One hundred and two lower eyelids of 68 patients were included in the study and the mean age at surgery was 47 years (median 47 ; range 20-82), with a preponderance of females (43 patients; 63\%). Most patients (49/68; $72 \%$ ) had thyroid eye disease, with idiopathic lower lid retraction (six patients; 9\%) and isolated previous squint surgery (four patients; $6 \%$ ) being the next commonest aetiologies (Table 1$)$. Forty one patients $(60 \%)$ had previously undergone one or more surgical procedures which could have influenced the lower eyelid position-in particular, 8/68 patients ( $12 \%$ patients; $16 / 102$ eyes) had undergone bilateral orbital decompression and 14/102 eyes had previous inferior rectus recession. Two patients had continued lower lid displacement despite previous placement of a scleral graft and many of the patients had previous minor procedures, such as lateral tarsorrhaphy, lateral canthal sling or medial canthoplasty.

All patients were unhappy with the appearance of lower lid displacement and most suffered ocular surface discomfort, with 18 patients $(27 \%)$ having marked lagophthalmos, and preoperative displacement ranged from

Table 1 Primary diagnosis for 68 patients undergoing hard palate mucosa grafting for displacement or retraction of the lower eyelid (102 lids)

\begin{tabular}{lll}
\hline Primary diagnosis & $\begin{array}{l}\text { Proportion of } \\
\text { patients }\end{array}$ & $\begin{array}{l}\text { Proportion of } \\
\text { eyelids }\end{array}$ \\
\hline Thyroid eye disease & $49 / 68(72 \%)$ & $76 / 102(74 \%)$ \\
Idiopathic retraction & $6 / 68(9 \%)$ & $9 / 102(9 \%)$ \\
Inferior rectus recession & $4 / 68(6 \%)$ & $6 / 102(6 \%)$ \\
Chronic progressive external ophthalmoplegia & $3 / 68(4 \%)$ & $5 / 102(5 \%)$ \\
Facial nerve palsy & $3 / 68(4 \%)$ & $3 / 102(3 \%)$ \\
Post-traumatic cicatricial eyelid malposition & $2 / 68(3 \%)$ & $2 / 102(2 \%)$ \\
High myopia & $1 / 68(2 \%)$ & $1 / 102(1 \%)$ \\
\hline
\end{tabular}

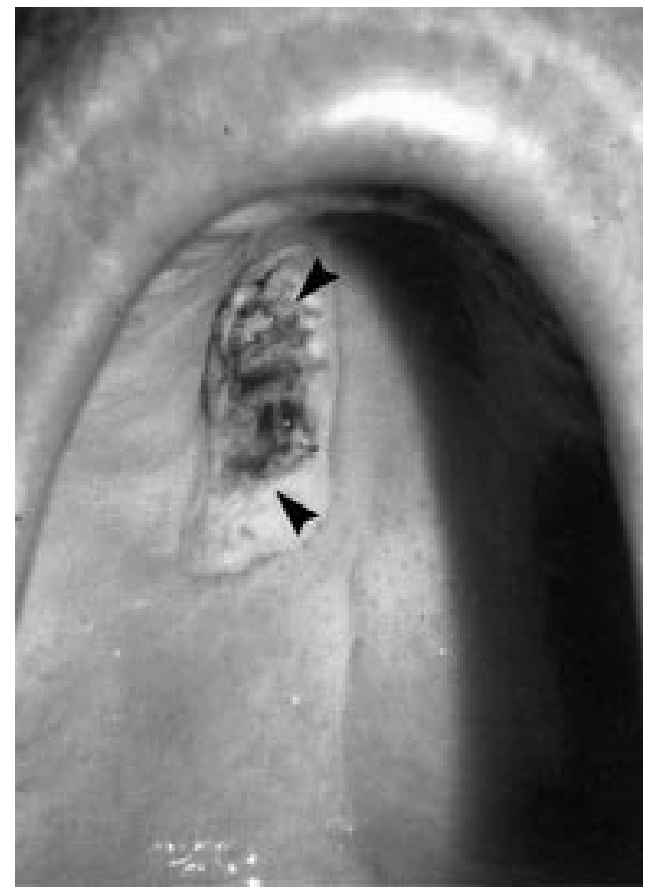

Figure 5 Advancing epithelial edge (arrows) at mucosal donor site a week after surgery.

1.5 to $6 \mathrm{~mm}$ (mean $3.1 \mathrm{~mm}$; median $3 \mathrm{~mm}$ ). Surgery was performed under a general anaesthetic in all but one case, usually by a consultant surgeon, and there were no intraoperative complications with either the donor or recipient sites, although two patients required placement of a haemostatic sponge to control donor site bleeding. The lower lid graft was augmented, in 25 lids, by simultaneous lateral canthal reattachment and, in some cases, a medial canthoplasty.

The mean postoperative scleral show at the lower limbus was $0.8 \mathrm{~mm}$, ranging from $1 \mathrm{~mm}$ overcorrection to $4 \mathrm{~mm}$ of residual show. Because of residual lower lid displacement, a poor outcome occurred in 15 lids (15\%) but, with an average follow up of 17 months (range 3-50 months), a "good" result was obtained in 40 eyelids (39\%) and an "acceptable" outcome in $47(46 \%)$.

Most patients were satisfied with the operation and the donor site generally healed rapidly by granulation (Fig 5). Postoperative complications did occur both at donor and recipient sites: Seven patients $(10 \%)$ had donor site haemorrhage at 7-12 days after surgery (mean 9 days), this typically being self limiting and settling with local pressure and systemic antibiotics. Two patients had intermittent soreness at the donor site. Ocular complications included chronic mucous discharge, passive oedema, stitch granuloma, cysts along the graft border, and a transient corneal abrasion (due to keratin) at 2 months after surgery. There were also three cases of bulky lower lids due to buckling (Fig 6) or excessive thickness of the graft.

\section{Discussion}

In most patients in this series, lower eyelid displacement was due to dysthyroid proptosis, the displacement being exacerbated by previous 

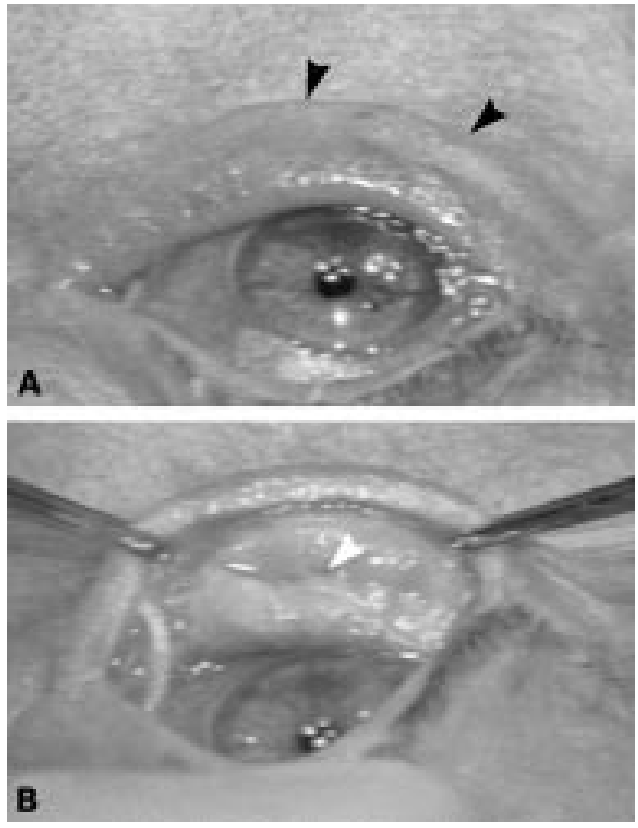

Figure 6 Thickening of the lower eyelid ( $A$; black arrows) due to palatal buckling as a result of fibrosis alongside the graft $(B ;$ white arrow). Viewed from above at surgery.

inferior retractor recession in some cases. Eyelid displacement increases the vertical palpebral aperture (with poor cosmesis, lagophthalmos, and ocular surface discomfort) and correction of lower lid displacement should be undertaken only after orbital decompression or extraocular muscle surgery has been performed.

Many procedures for correction of lower lid displacement have been proposed: non-graft techniques (free tenotomy, inferior retractor recession, or extirpation of the lower lid retractors ${ }^{25-27}$ ) may be used for mild cases, but are ineffective where lower lid displacement is moderate or severe. Several donor materials have been used as spacers for more severe displacement of the lower eyelid, including autogenous grafts (for example, hard palate mucosa, tarsus, ear cartilage), homologous grafts (for example, sclera, fascia lata), or synthetic spacer materials (for example, poly(tetrafluoroethylene), polyester mesh, thin porous polyethylene sheet). The large number of materials described suggests that the optimum spacer remains controversial.

Homologous grafts carry a risk of disease transmission, which may be unacceptable to some patients. Donor sclera is, however, useful for lower lid lengthening, ${ }^{35628}$ but tends to be associated with recurrent retraction due to graft absorption and fibrosis ${ }^{3629}$; although conjunctival closure over a scleral graft might reduce its absorption, this closure might also reduce the effect of the retractor recession. Ear cartilage grafts, fairly easily harvested, ${ }^{30}$ may impede downgaze as a result of a relatively immobile lower eyelid. Free tarsal grafting ${ }^{31}$ is limited by the amount of donor material available and by the fact that harvest from the upper eyelid donor site may exacerbate pre-existing
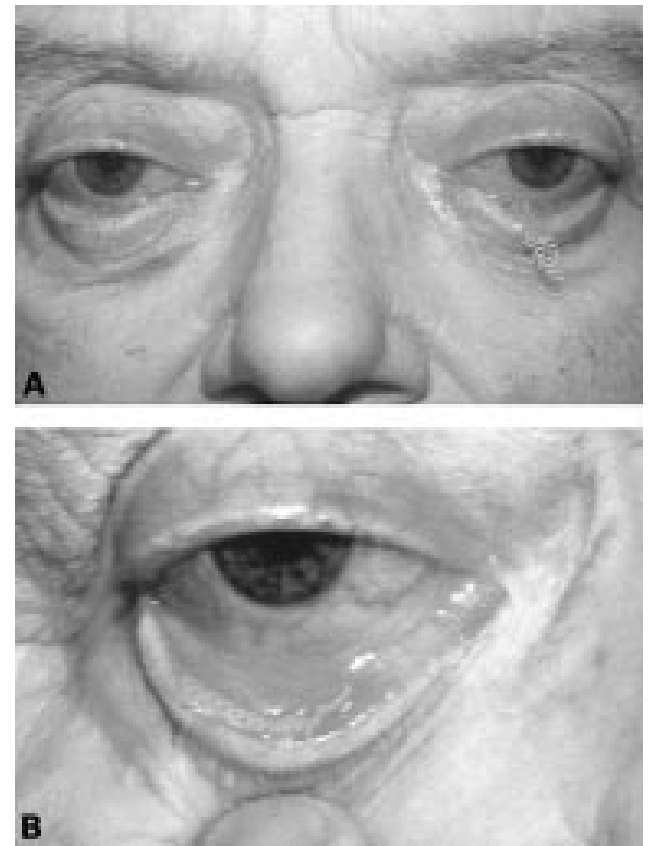

Figures 7 Patient referred with bilateral extrusion of polyester mesh $(A)$, which had been used as a lower lid "spacer" (B).

upper eyelid retraction. Synthetic materials have a significant risk of erosion and extrusion (Fig 7).

Autogenous hard palate mucosa approximates to lower lid tarsus in terms of contour, thickness and stiffness, has a mucosal surface, and has no risk of rejection. In this series of patients we have found palatal mucosa, when used as a spacer, gave an acceptable result in $85 \%$ of eyelids. Although analysis of eyelids with a "poor" result did not reveal a common factor, the degree of preoperative lower lid retraction was slightly greater in this group$3.6 \mathrm{~mm}$ (range 3-6 $\mathrm{mm}$ ) compared with 2.9 $\mathrm{mm}$ in the successful group.

Ophthalmic complications were uncommon and are generally managed easily. Seven patients, however, had donor site secondary haemorrhage, a problem well documented in the literature ${ }^{32}$ and which may be prevented by use of protective dental pastes and custom made acrylic stents. ${ }^{33}$ Such stents also have the advantage that, should postoperative bleeding occur, pressure can be applied to the donor site. Other reported complications include oro-nasal fistula and compromised healing due to oral candidiasis, ${ }^{24}$ both resolving with appropriate treatment.

A hard palate mucosal graft is, therefore, an effective spacer for elevating the majority of displaced lower eyelids, although the main disadvantage (compared with other autogenous materials) is donor site morbidity, which may be minimised by meticulous surgical technique and appropriate postoperative care. Although harvesting of palatal mucosa is usually straightforward, the results may be unsatisfactory if a surgeon is not totally familiar with the palatal anatomy and the individual variations that can exist. 
1 Karesh JW, Fabrega MA, Rodrigues MM, et al. Polytetrafluoroethylene as an interpositional graft material for the correction of lower eyelid retraction. Ophthalmology 1989;96:419-23.

2 Downes RN, Jordan $\mathrm{K}$. The surgical management of dysthyroid related eyelid retraction using mersilene mesh Eye 1989;3:385-90.

3 Dryden RM, Soll DB. The use of scleral transplantation in cicatricial entropion and eyelid retraction. Trans Am Acad Ophthalmol Otolaryngol 1977;83:669-76.

4 Doxanas MT, Dryden RM. The use of sclera in the treatment of dysthyroid eyelid retraction. Ophthalmology 1981;88:887-94.

5 Mourits MP, Koorneef L. Lid lengthening by sclera interposition for eyelid retraction in Graves' ophthalmopathy. Br f Ophthalmol 1991;75:344-7.

6 Olver JM, Rose GE, Khaw PT, et al. Correction of lower eyelid retraction in thyroid eye disease: a randomised controlled trial of retractor tenotomy with adjuvant antimetabolite versus scleral graft. Br 7 Ophthalmol 1998;82:17480.

7 Siegel RJ. Palatal grafts for eyelid reconstruction. Plast Reconstruct Surg 1985;76:411-16.

8 Patel BC, Patipa M, Anderson RL, et al. Management of postblepharoplasty lower eyelid retraction with hard palate grafts and lateral tarsal strip. Plast Reconstruct Surg 1997;99:1251-60.

9 Kersten RC, Kulwin DR, Levartovsky S, et al. Management of lower-lid retraction with hard-palate mucosa grafting. Arch Ophthalmol 1990;108:1339-43.

10 Mannor GE, Mathers WD, Wolfley DE, et al. Hard-palate mucosa graft in Stevens-Johnson syndrome. Am f Ophthalmol 1994;118:786-91.

11 Yoshimura Y, Nakajima T, Yoneda K. Use of the palatal mucosal graft for reconstruction of the eye socket. CranioMaxillo-Facial Surg 1995;23:27-30.

12 Holck D, Foster JA, Dutton JJ, et al. Hard palate mucosal grafts in the treatment of the contracted socket. Ophthalmic grafts in the treatment of the contract

13 Kim YD, Goldberg RA, Shorr N, et al. Management of exposed hydroxyapatite orbital implants. Ophthalmology 1994;101:1709-15.

14 Hatoko $M$, Tada $H$, Shirai T. Usefulness of hard palate mucosa graft as nasal lining in alar reconstruction. Plast Reconstruct Surg 1995;95:390-5.

15 Vecchione TR. Palatal grafts for lip reconsruction. Ann Plast Surg 1983;10:301-5.

16 Wobig JL, Loff HJ, Dailey RA. Vertical eyelid shortening. In Levine MR, ed. Manual of oculoplastic surgery. 2nd ed. Boston: Butterworth-Heinemann, 1996:143-4.
17 McMinn RMH. Head and neck. In: Last's anatomy regional and applied. 8th ed. Oxford: Churchill Livingstone, 1990:479-80.

18 Studer SP, Allen EP, Rees TC, et al. The thickness of masticatory mucosa in the human hard palate and tuberosity as potential donor sites for ridge augmentation procedures. 7 Periodontal 1997;68:145-51.

19 Reiser GM, Bruno JF, Mahan PE, et al. The subepithelial connective tissue graft palatal donor site: anatomic considcor surgeons. Int f Periodon Rest Dent 1996;16:130-

20 Ajmani ML. Anatomical variation in position of the greater palatine foramen in the adult skull. F Anatomy 1994;184: 635-7.

21 Nery F, Corn H, Eisenstein IL. Palatal exostoses in the molar region. F Periodontal 1977;48:633-6.

22 Beatty RL, Harris G, Bauman GR, et al. Intraoral palatal mucosal graft harvest. Ophthalmic Plast Reconstr Surg 1993; 9:120-4.

23 Wiggins NE, Engel LD. Free palatal mucosa grafts. Oral Surg 1973;35:35-40.

24 Kim JW, Kikkawa DO, Lemke BN. Donor site complications of hard palate mucosal grafting. Ophthalmic Plast Reconstr Surg 1997;13:36-9.

25 Henderson JW. Relief of eyelid retraction. A surgical procedure. Arch Ophthalmol 1965;74:205-16.

26 Waller RR. Lower eyelid retraction: management. Ophthalmic Surg 1978;9:41-7.

27 Holds JB, Anderson RL, Thiese SM. Lower eyelid retraction: a minimal incision surgical approach to retractor lysis. Ophthalmic Surg 1990;21:767-71.

28 Shorr N, Seiff SR. The four stages of surgical rehabilitation of the patient with dysthyroid ophthalmopathy. Ophthalmology 1986;93:476-83.

29 Harvey JT, Anderson RL. The aponeurotic approach to eyelid retraction. Ophthalmology 1981;88:513-24.

30 Bayliss HI, Pearman KI, Fett DR, et al. Autogenous auricular cartilage grafting for lower eyelid retraction. Ophthalmic Plast Reconstr Surg 1985;1:23-7.

31 Obear MF, Smith B. Tarsal grafting to elevate the lower lid margin. Am 7 Ophthalmol 1965;59:1088-90.

32 Mauriello JA, Wasserman B, Allee S, et al. Moulded acrylic mouthguard to control bleeding at the hard palate graft site after eyelid reconstruction. Am f Ophthalmol 1992;113: $342-4$

33 Shorr N, Enzer YR. Moulded acrylic mouthguard to control bleeding at the hard palate graft site after eyelid reconstruction (letter). Am F Ophthalmol 1992;114:779-80. 